\title{
Predictive sonographic features for differentiation of breast fibroepithelial tumors: fibroadenoma versus phyllodes tumor
}

Ga Young Lee ${ }^{1}$, Gi Won Shin ${ }^{1}$, Ha Young Park ${ }^{2}$, Hye Kyoung Yoon ${ }^{2}$, Tae Hyun Kim ${ }^{3}$, Anbok Lee $^{3}$, Young Jin Heo ${ }^{1}$, Yoo Jin Lee ${ }^{1}$, Ji-Yeon Han ${ }^{1}$, Young Mi Park *1

${ }^{1}$ Department of Radiology,

${ }^{2}$ Department of Pathology,

${ }^{3}$ Department of Surgery,

Busan Paik Hospital, Inje University College of Medicine, Busan, South Korea

Correspondence: Dr Young Mi Park, Department of Radiology, Busan Paik Hospital, Inje University College of Medicine, Busan, South Korea

Email: nanbarkym@hanmail.net

Submitted: 22 Jan 2021; Accepted: 23 April 2021

Contributors: All authors designed the study. All authors acquired the data. GYL, GWS, YJH, YMP analysed the data. All authors drafted the manuscript. GYL, GWS, JYH, YMP critically revised the manuscript for important intellectual content. All authors had full access to the data, contributed to the study, approved the final version for publication, and take responsibility for its accuracy and integrity.

Conflicts of Interest: All authors have disclosed no conflicts of interest.

Funding/Support: This research received no specific grant from any funding agency in the public, commercial, or not-for-profit sectors.

Ethics Approval: This study was approved by the Institutional Review Board of Busan Paik Hospital. The requirement for informed consent was waived.

This article has undergone peer review and has been accepted for publication in Hong Kong Journal of Radiology. This Author Accepted Manuscript has not been copyedited, typeset, or proofread, and this version may differ from the final published Version of Record.

Please cite this article as an 'Accepted Article'. https://doi.org/10.12809/hkjr2117333 


\section{Abstract}

\section{Objective}

Fibroepithelial tumors (FETs) of the breast are benign tumors, which include fibroadenoma (FA) and phyllodes tumor (PT). Although they show different biological behaviors, differentiation using imaging features or core needle biopsy is challenging. Therefore, we evaluated sonographic features that could be useful for differentiating FA and PT and the diagnostic accuracy of core needle biopsy for differentiating breast FETs.

\section{Methods}

A total of 121 patients with 125 lesions diagnosed as fibroepithelial tumors on US-guided core needle biopsy from March 2017 to April 2020 were studied. Among them, sonographic features of 68 lesions were analyzed retrospectively. Clinicopathologic results of core needle biopsy and surgical excision were reviewed using electronic medical records.

\section{Results}

Tumor size, echogenicity, presence of an internal cleft, vascularity, and elasticity showed significant differences between FA and PT. A large tumor size $(\geq 3 \mathrm{~cm})$, presence of an internal cleft, and hard elasticity were common sonographic features in PTs. Core needle biopsy revealed similar pathologic results with surgical excision performed in 61 cases of 68 cases $(89 \%)$.

\section{Conclusion}

Ultrasonographic features can be useful imaging factors for differentiating FA and PT. Moreover, US-guided core needle biopsy can replace surgical excision with a high diagnostic accuracy in some cases.

Key Words : breast, fibroadenoma, phyllodes tumor 


\section{INTRODUCTION}

Fibroepithelial tumors of the breast, including fibroadenoma (FA) and phyllodes tumor (PT), are composed of a biphasic proliferation of both epithelial and stromal components. ${ }^{1}$ The distinction between FA and PT is clinically important. While FAs generally regress with age and can be safely followed without further investigations, PTs continue to grow requiring wide local excision to prevent local recurrence. ${ }^{2-4}$ The risk of local recurrence in PTs ranges from $17 \%$ in benign PT to $27 \%$ in malignant PT, with metastasis occurring in approximately $25 \%$ of malignant PTs. ${ }^{5}$ Most recurrent PTs are histologically similar to initial tumors; however, in upto $26 \%$ of initial benign PTs, there is a risk of recurrence as borderline or malignant PTs. ${ }^{6,7}$

Although the two disease entities show different biological behaviors, differentiating them using imaging features or core needle biopsy is challenging. ${ }^{8,9}$ Histologically, PT is usually distinguished from FA based on the presence of hypercellular stroma that show leaf-like projection into the stroma. However, a definite distinction with core biopsy is difficult owing to the small sample size and because hypercellularity also can be present in juvenile FAs and in the breast tissue in women receiving hormone replacement therapy. ${ }^{10-12}$

If the disease entities can be differentiated using image findings and core needle biopsy results, unnecessary surgical excision can be avoided. Therefore, we evaluated the sonographic features that could differentiate FA and PT and the diagnostic accuracy of core needle biopsy (CNB) for the differentiation of breast FETs.

\section{METHODS}

\section{Patient population}

This retrospective study was approved by the Institutional Review Board of our hospital, and the requirement for informed consent was waived. Three radiologists retrospectively reviewed the medical and imaging records of patients diagnosed with breast fibroepithelial tumors on ultrasound (US)-guided CNB. From March 2017 to April 2020, 125 lesions in 121 patients were confirmed to be fibroepithelial tumors. Among them, cases without surgical excision after CNB ( $n=46)$, cases with US-guided vacuum assisted biopsy after CNB $(n=8)$, cases confirmed as another lesion (sclerosing adenosis) $(\mathrm{n}=1)$, and cases confirmed as both FA and PT $(n=2)$ were excluded. Among 46 cases without surgical excision, 16 cases were not available for imaging follow up after core needle biopsy. And the range of follow up interval 
for 30 cases was $2 \sim 48$ months. There were 5 cases which showed decrease of lesion size during follow up period. Other 25 cases showed no interval change during imaging follow up period. Finally, a total 68 cases of 65 patients were included.

\section{Data acquisition and data analysis}

Breast ultrasonography was performed using a 15-4 MHz (Aixplorer, SuperSonic Imagine, Aix-en-Provence, France) or 5-12 MHz (iU22; Philips Healthcare, Bothell, WA, USA) linear array transducer. The sonographic features of 68 lesions were analyzed independently and retrospectively by three breast dedicated radiologists, with 1, 5 and 20 years of experience, according to the American College of Radiology (ACR) Breast Imaging Reporting and Data System (BI-RADS) Atlas ( $5^{\text {th }}$ edition). ${ }^{13}$ The maximum elasticity value (Emax) of shear-wave elastography was categorized into hard $($ Emax $\geq 144 \mathrm{kPa})$, intermediate $(72<$ Emax $<144)$ and soft elasticity $(E \max \leq 72){ }^{14}$ US-guided CNB was performed by using a 14-gauge cutting needle using a freehand technique, and five core specimens were retrieved from each lesion. Clinicopathologic results of the CNB and surgical excision were reviewed using electronic medical records. The pathologic reports of all the CNBs included the suggestion of the pathologists, such as FET favoring FA or PT, and we analyzed the concordance between the pathologists' suggestion in CNB and the pathologic results of surgical excision.

\section{Statistical analysis}

Comparison of the categorical variables between FA and PT, benign and borderline/malignant PT was performed using the chi-square and Fisher's exact tests. The level of significance was set at 0.05 for all tests. The statistical analysis was conducted using SPSS statistics v. 26.0 (IBM, Armonk, NY, USA).

\section{RESULTS}

Among the 68 lesions analyzed in this study, the pathologic results of surgical excision revealed 32 FAs and 36 PTs. The mean age of patients with FA was 41.7 years (range, 20-68 years) and those with PT was 39.3 years (range, 18-52 years). Among the 36 cases of PTs, the histologic subtypes were benign in 32 cases, $(88.9 \%)$ and borderline and malignant PT in two 
cases each $(5.6 \%)$.

Regarding sonographic features, tumor size $(\mathrm{p}=0.003)$, echogenicity $(\mathrm{p}=0.002)$, presence of an internal cleft $(p=0.017)$, vascularity $(p=0.032)$, and elasticity $(p=0.028)$ showed significant differences between FA and PT [Table 1]. FAs were frequently small $(<3 \mathrm{~cm})$, hypoechoic masses with soft or intermediate elasticity and without an internal cleft [Figure 1]. PTs were significantly larger than FAs ( $\mathrm{p}=0.003)$ [Figure 2]. Among the PTs, 11 lesions were larger than $3 \mathrm{~cm}$ in size $(11 / 36,30.6 \%)$, while only one lesion $(1 / 32,3.1 \%)$ was larger than $3 \mathrm{~cm}$ among FAs. The echogenicity in PTs was frequently heterogeneous $(\mathrm{PT}=13.9 \%$ vs. $\mathrm{FA}=3.1 \%)$ and showed a complex cystic and solid pattern $(\mathrm{PT}=19.4 \%$ vs. $\mathrm{FA}=0 \%)$ with significant difference. The presence of an internal cleft $(\mathrm{PT}=44.4 \%$ vs. $\mathrm{FA}=15.6 \%)$, internal vascularity $(\mathrm{PT}=75 \%$ vs. $\mathrm{FA}=43.8 \%)$, and hard elasticity $(\mathrm{PT}=22.2 \%$ vs. $\mathrm{FA}=3.1 \%)$ were more frequently observed in PT than in FA with a significant difference [Figure 3].

Other sonographic features including shape, orientation, margin, posterior features, associated calcifications, distortion, ductal change, skin change, and edema showed no significant differences between FA and PT.

The pathologic results of CNB were similar to that of surgical excision in 61 cases $(61 / 68$, 89.7\%). Seven cases with discordant results were all reported as FA on CNB and benign PT on surgical excision.

Subgroup analysis for analysis imaging features between benign and borderline/malignant PT was done. The lesion size $(\geq 3 \mathrm{~cm})[p=0.002]$, presence of internal cleft [ $p=0.031]$ and echogenicity [ $[\mathrm{p}=0.029]$ showed significant difference between benign and borderline/malignant PT. The larger tumor size $(\geq 3 \mathrm{~cm})$, presence of internal cleft and complex echogenicity were more frequently observed in borderline/malignant PT than benign PT with a significant difference.

\section{DISCUSSION}

In this study, we evaluated sonographic features that could be helpful for the differentiation of FA and PT. Differentiation of FAs and PTs using imaging features or core needle biopsy has been challenging to radiologists and pathologists. ${ }^{8,9}$ Moreover, as US-guided CNB specimens only reveal a part of the total lesion and as two disease entities can have overlapping histologic features, pathologists often are unable to make a definitive distinction between FA and PT. ${ }^{15-17}$ 
We found that a large tumor with heterogeneous/complex cystic and solid echogenicity and the presence of an internal cleft on grayscale ultrasonography were significant findings of PT, in accordance with previous studies. ${ }^{3,8-10}$ Duman et al. reported that FAs were smaller than PTs and had a homogeneously hypoehoic mass, while PTs showed heterogeneous/complex cystic and solid echogenicity. The presence of internal cystic lesions was significantly more commonly detected in PTs than in FAs. ${ }^{8}$ The presence of internal cystic lesions and clefts is regarded as a useful US feature to diagnose PTs. ${ }^{3,9,10,18}$ In a study by Wiratkapun et al., the presence of cystic spaces and clefts within the solid mass was significantly associated with PTs on both univariate $(p<0.001)$ and multivariate analyses (cystic space; $p=0.032$, clefts; $\mathrm{p}=0.006) .{ }^{3}$ This finding is related to microscopic features of PTs, with a prominent stromal proliferation into the epithelial lined spaces, forming a slit-like space or a leaf-like pattern (Figure 3D, 3E) ${ }^{19}$

Posterior acoustic enhancement was also found to be an important sonographic feature of PTs in some studies. ${ }^{9,20}$ In our study, posterior acoustic enhancement was more frequently observed in PTs, but without significant difference $(\mathrm{PT}=44.4 \%$ vs $\mathrm{FA}=25 \%, \mathrm{p}=0.161)$.

Color Doppler ultrasound and elastography have been investigated as ancillary tools to improve the diagnostic accuracy of conventional B-mode ultrasound for breast lesions. Kim et al. analyzed the color Doppler ultrasonography and shear-wave elastography features of fibroepithelial lesions and reported that PTs tend to have higher stiffness and vascularity than FAs. The median $\mathrm{E}_{\max }$ was significantly higher in PTs than in FAs (76.7 vs. $\left.21.0 \mathrm{kPa}, \mathrm{p}<0.01\right)$, and a high vascularity (equal to or greater than two vessels) on color Doppler US was more frequent in PTs than in FAs $(\mathrm{p}<0.01) .{ }^{21}$ Similar to that reported in a previous study, internal vascularity $(\mathrm{PT}=75 \%$ vs. $\mathrm{FA}=43.8 \%, \mathrm{p}=0.032)$ and hard elasticity $(\mathrm{PT}=22.2 \%$ vs. $\mathrm{FA}=$ $3.1 \%, \mathrm{p}=0.028$ ) were more frequently seen in PTs than FAs in this study. Histologically, on shear-wave elastography, the more abundant cellular stroma in PTs than in FAs is associated with the hard elasticity of PTs. ${ }^{15,19}$

A definitive distinction between FA and PT is challenging, particularly with CNB specimens. ${ }^{15-17}$ Based on the degree of stromal hypercellularity, stromal overgrowth, nuclear atypia, mitoses counts, and the amount of stroma relative to the epithelium and infiltrative borders of the tumor, PTs are differentiated from FAs and categorized into benign, borderline, and malignant lesions. ${ }^{3,12,15}$ However, the representation of these features in CNB has not been proven to be reliably adequate for pathologists to make a definitive diagnosis of PT using 
CNB. ${ }^{12}$ Wiratkapun et al. found a high concordance between pathologists' suggested diagnosis of FA or PT from CNB specimens and the surgical pathology after excision $(\mathrm{p}<0.001){ }^{3}$ Komenaka et al. also found similar results, showing an $83 \%$ positive predictive value of diagnosis for FETs using CNB. ${ }^{22}$ Our study showed a high diagnostic accuracy of the pathologic results of $\mathrm{CNB}$ for differentiation between FA and PT, which is similar to the findings with previous studies. ${ }^{3,22}$ If result for core needle biopsy was FET favoring FA and ultrasonography showed relatively suggestive features for FA rather than PT, we can consider the omission for surgical excision and recommend imaging and clinical follow up. According to the literature review, large size, irregular shape, indistinct margin and complex echogenicity can be the helpful factors for differentiation between benign and borderline/malignant PT. ${ }^{23}$ In our study, for subgroup analysis, large size $(\geq 3 \mathrm{~cm})$, presence of internal cleft and complex echogenicity showed significant difference between benign and borderline/malignant PT. Although this study included only four cases of borderline and malignant PTs, results were relatively similar to previous reports.

There are several limitations to our study. First, this study had a retrospective single-center design. Second, the small sample size of enrolled masses could have prevented an accurate statistical analysis, with a degree of selection bias as only excised lesions were included. Further investigations with a large sample size are needed. Third, the analysis in this study was based only on ultrasonographic findings.

\section{CONCLUSION}

In conclusion, ultrasonographic features can be helpful imaging factors for differentiating FAs and PTs. Imaging features such as a large tumor size, heterogeneous or complex cystic and solid echogenicity, presence of an internal cleft, internal vascularity, and hard elasticity were significant findings in PT. Moreover, US-guided CNB may replace surgical excision with high diagnostic accuracy in some cases. 


\section{References}

1. Lakhani SR, Ellis IO, Schnitt S, Tan PH, van de Vijver M. WHO Classification of Tumours of the Breast. 2012.

2. Karim RZ, O'Toole SA, Scolyer RA, Cooper CL, Chan B, Selinger C, et al. Recent insights into the molecular pathogenesis of mammary phyllodes tumours. Journal of Clinical Pathology. 2013;66(6):496-505.

3. Wiratkapun C, Piyapan P, Lertsithichai P, Larbcharoensub N. Fibroadenoma versus phyllodes tumor: distinguishing factors in patients diagnosed with fibroepithelial lesions after a core needle biopsy. Diagnostic and Interventional Radiology. 2014;20(1):27.

4. Montagna G, Ng CK, Vlajnic T, Paradiso V, Dellas S, Reina H, et al. Fibroepithelial Breast Lesion: When Sequencing Can Help to Make a Clinical Decision. A Case Report. Clinical breast cancer. 2019;19(1):e1-e6.

5. Zhang Y, Kleer CG. Phyllodes tumor of the breast: histopathologic features, differential diagnosis, and molecular/genetic updates. Arch Pathol Lab Med. 2016;140(7):665-71.

6. Xiao M, Zhu Q, Jiang Y, Li J, Wang H, Zhang J, et al. Local recurrent phyllodes tumors of the breast: clinical and sonographic features. J Ultrasound Med. 2015;34(9):1631-8.

7. Barrio AV, Clark BD, Goldberg JI, Hoque LW, Bernik SF, Flynn LW, et al. Clinicopathologic features and long-term outcomes of 293 phyllodes tumors of the breast. Ann Surg Oncol. 2007;14(10):2961-70.

8. Duman L, Gezer NS, Balcı P, Altay C, Başara I, Durak MG, et al. Differentiation between phyllodes tumors and fibroadenomas based on mammographic sonographic and MRI features. Breast Care. 2016;11(2):123-7.

9. Yilmaz E, Sal S, Lebe B. Differentiation of phyllodes tumors versus fibroadenomas: mammographic and sonographic features. Acta radiologica. 2002;43(1):34-9.

10. Bode M, Rissanen T, Apaja-Sarkkinen M. Ultrasonography and core needle biopsy in the differential diagnosis of fibroadenoma and tumor phyllodes. Acta radiologica. 2007;48(7):708-13.

11. Shousha S. Issues in the interpretation of breast core biopsies. Int J Surg Pathol. 2003;11(3):167-76.

12. Dillon MF, Quinn CM, McDermott EW, O’Doherty A, O’Higgins N, Hill AD. Needle core biopsy in the diagnosis of phyllodes neoplasm. Surgery. 2006;140(5):779-84.

13. Radiology ACo, D'Orsi CJ. ACR BI-RADS Atlas: Breast Imaging Reporting and Data 
System; Mammography, Ultrasound, Magnetic Resonance Imaging, Follow-up and Outcome Monitoring, Data Dictionary: ACR, American College of Radiology; 2013.

14. Lee SH, Chang JM, Cho N, Koo HR, Yi A, Kim SJ, et al. Practice guideline for the performance of breast ultrasound elastography. Ultrasonography. 2014;33(1):3.

15. Jacobs TW, Chen Y-Y, Guinee Jr DG, Holden JA, Cha I, Bauermeister DE, et al. Fibroepithelial lesions with cellular stroma on breast core needle biopsy: Arethere predictors of outcome on surgical excision? American journal of clinical pathology. 2005;124(3):342-54. 16. Resetkova E, Khazai L, Albarracin CT, Arribas E. Clinical and radiologic data and core needle biopsy findings should dictate management of cellular fibroepithelial tumors of the breast. The breast journal. 2010;16(6):573-80.

17. Choi J, Koo JS. Comparative study of histological features between core needle biopsy and surgical excision in phyllodes tumor. Pathology international. 2012;62(2):120-6.

18. Chao TC, Lo YF, Chen SC, Chen MF. Sonographic features of phyllodes tumors of the breast. Ultrasound in Obstetrics and Gynecology: The Official Journal of the International Society of Ultrasound in Obstetrics and Gynecology. 2002;20(1):64-71.

19. Gary M, Niu Y, Shi H-J. Phyllodes tumor of the breast: an update. Breast cancer. 2010;17(1):29.

20. Buchberger W, Strasser K, Heim K, Müller E, Schröcksnadel H. Phylloides tumor: findings on mammography, sonography, and aspiration cytology in 10 cases. AJR American journal of roentgenology. 1991;157(4):715-9.

21. Kim GR, Choi JS, Han B-K, Ko EY, Ko ES, Hahn SY. Combination of shear-wave elastography and color Doppler: Feasible method to avoid unnecessary breast excision of fibroepithelial lesions diagnosed by core needle biopsy. Plos one. 2017;12(5):e0175380.

22. Komenaka IK, El-Tamer M, Pile-Spellman E, Hibshoosh H. Core needle biopsy as a diagnostic tool to differentiate phyllodes tumor from fibroadenoma. Arch Surg. 2003;138(9):987-90.

23. Shin GW, Park YM, Park JH, Kim HJ, Choo HJ, Baek HJ, et al. Phyllodes tumour of the breast: differentiation of histological grade by ultrasonography. Hong Kong J Radiol. 2016;22:107-13. 


\section{Figures}

Figure 1. Fibroadenoma in a 31-year-old women with palpable lump in the right breast. (a, b, c) Ultrasonograms show a $2.2 \mathrm{~cm}$ sized oval, circumscribed, hypoechoic mass with mild internal vascularity and intermediate elasticity $\left(\mathrm{E}_{\max }=70.4 \mathrm{kPa}\right)$. (d, e) Microscopic examinations of US-guided CNB (H\&E stain, x40) and surgical excision (H\&E stain, x40) show periductal (blank arrowheads) and stromal (arrowhead) hyalinization, suggestive for fibroadenoma.

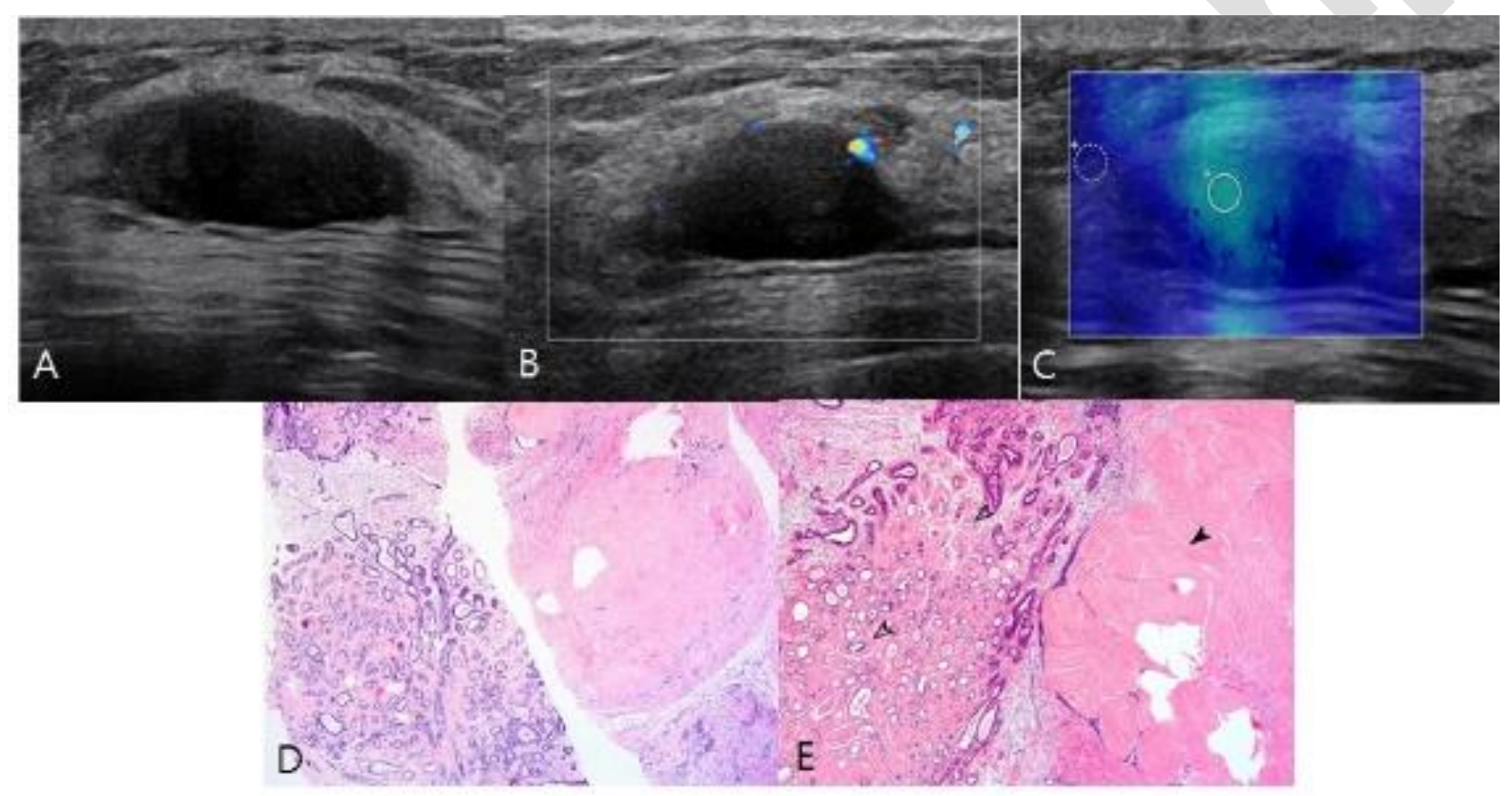


Figure 2. Benign phyllodes tumor in a 33-year-old women with palpable lump in the right breast. (a, b, c) Ultrasonograms show about a $9 \mathrm{~cm}$ sized oval, circumscribed, hypoechoic mass with internal cystic cleft (arrows), internal vascularity. (d, e) Microscopic examinations of USguided CNB (H\&E stain, x40) and surgical excision (H\&E stain, x400) show characteristic leaf-like structures, suggestive for phyllodes tumor.

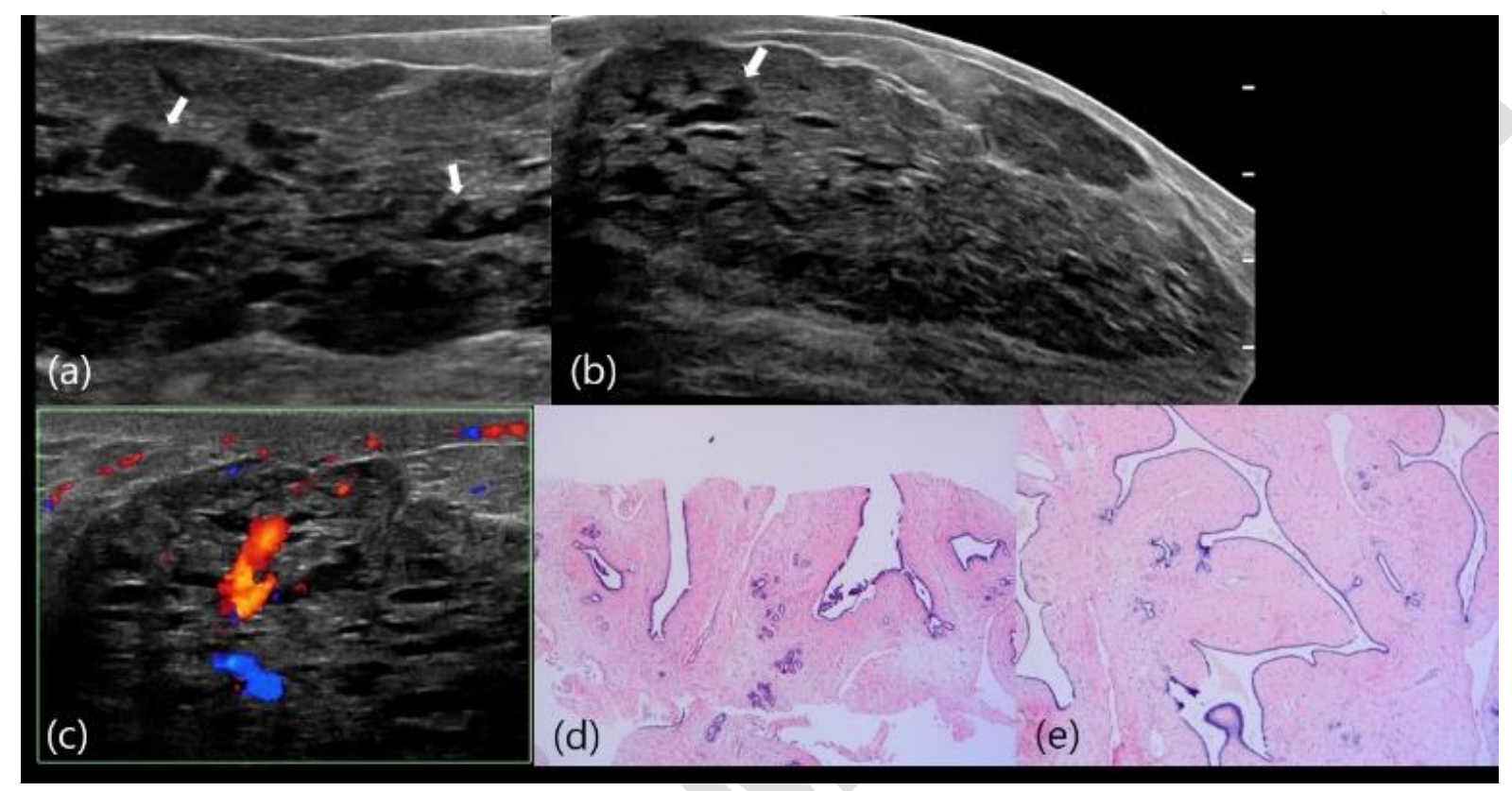


Figure 3. Benign phyllodes tumor in a 35-year-old women with palpable lump in the left breast. (a, b, c) Ultrasonograms show a $2.5 \mathrm{~cm}$ sized oval, circumscribed, hypoechoic mass with internal cystic cleft (arrow), internal vascularity and hard elasticity $\left(E_{\max }=85.2 \mathrm{kPa}\right)$. (d, e) Microscopic examinations of US-guided CNB (H\&E stain, x100) and surgical excision (H\&E stain, x100) show mildly increased cellularity and cellular atypia in stroma, suggestive for benign phyllodes tumor.

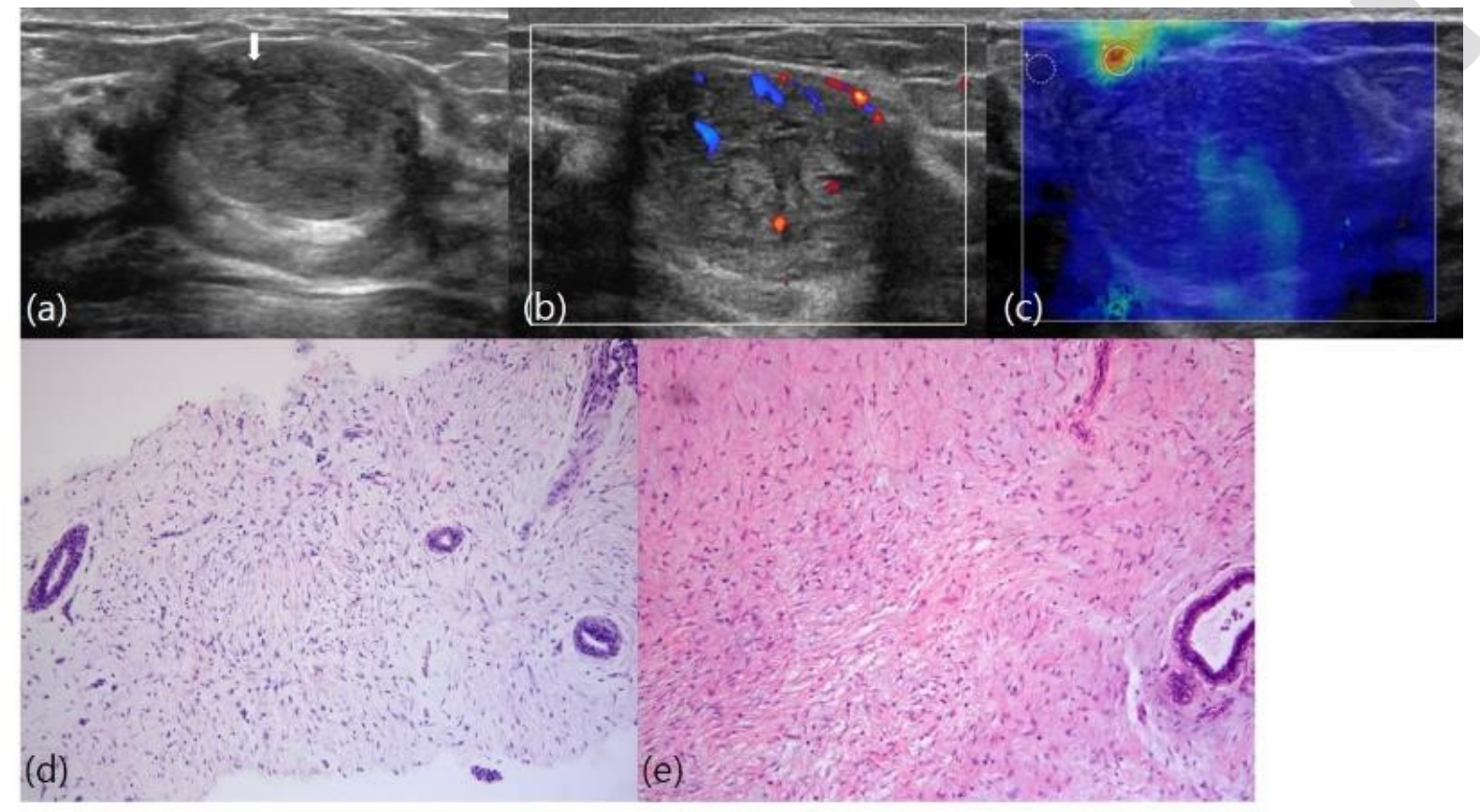




\section{Tables}

Table 1. Ultrasonographic findings in fibroadenomas and phyllodes tumors

\begin{tabular}{|c|c|c|c|c|}
\hline \multicolumn{2}{|c|}{ Lesion characteristics } & $\begin{array}{l}\text { Fibroadenoma } \\
\quad(n=32)\end{array}$ & $\begin{array}{l}\text { Phyllodes } \\
\text { tumor }(n=36)\end{array}$ & P-value \\
\hline \multirow[t]{2}{*}{ Size } & $<3 \mathrm{~cm}$ & $31(96.9 \%)$ & $25(69.4)$ & \multirow{2}{*}{$0.003^{+}$} \\
\hline & $\geq 3 \mathrm{~cm}$ & $1(3.1 \%)$ & $22(30.6 \%)$ & \\
\hline \multirow{3}{*}{ Shape } & Oval & $28(87.5 \%)$ & $28(77.8 \%)$ & \multirow{3}{*}{$0.504^{*}$} \\
\hline & Round & $1(3.1 \%)$ & $1(2.8 \%)$ & \\
\hline & Irregular & $3(9.4 \%)$ & $7(19.4 \%)$ & \\
\hline \multirow[t]{2}{*}{ Orientation } & Parallel & $35(100 \%)$ & $32(89 \%)$ & \multirow{2}{*}{$0.116^{+}$} \\
\hline & Non-parallel & 0 & $4(11 \%)$ & \\
\hline \multirow[t]{4}{*}{ Echogenecity } & Hypoechoic & $31(96.9 \%)$ & $21(58.3 \%)$ & \multirow{4}{*}{$0.002^{*}$} \\
\hline & Heterogeneous & $1(3.1 \%)$ & $5(13.9 \%)$ & \\
\hline & Isoechoic & 0 & $3(8.3 \%)$ & \\
\hline & $\begin{array}{l}\text { Complex cystic and } \\
\text { solid }\end{array}$ & 0 & $7(19.4 \%)$ & \\
\hline \multirow[t]{5}{*}{ Margin } & Circumscribed & $18(56.3 \%)$ & $21(58.3 \%)$ & \multirow{5}{*}{$0.380^{*}$} \\
\hline & Indistinct & $7(21.9 \%)$ & $9(25 \%)$ & \\
\hline & Angular & 0 & $2(5.6 \%)$ & \\
\hline & Microlobulated & $7(21.9 \%)$ & $4(11.1 \%)$ & \\
\hline & Spiculated & 0 & 0 & \\
\hline \multirow[t]{2}{*}{ Internal cleft } & Present & $5(15.6 \%)$ & $16(44.4 \%)$ & \multirow{2}{*}{$0.017^{+}$} \\
\hline & Absent & $27(84.4 \%)$ & $20(55.6 \%)$ & \\
\hline \multirow{3}{*}{ Vascularity } & No & $10(31.3 \%)$ & $5(13.9 \%)$ & \multirow{3}{*}{$0.032^{*}$} \\
\hline & Internal vascularity & $14(43.8 \%)$ & $27(75 \%)$ & \\
\hline & Vessels in rim & $8(25 \%)$ & $4(11.1 \%)$ & \\
\hline \multirow[t]{4}{*}{ Elasticity } & Soft & $12(37.5 \%)$ & $7(19.4 \%)$ & \multirow{4}{*}{$0.028^{*}$} \\
\hline & Intermediate & $9(28.1 \%)$ & $5(13.9 \%)$ & \\
\hline & Hard & $1(3.1 \%)$ & $8(22.2 \%)$ & \\
\hline & No result & $10(31.3 \%)$ & $16(44.4 \%)$ & \\
\hline \multirow{3}{*}{$\begin{array}{l}\text { Posterior } \\
\text { feature }\end{array}$} & No & $23(71.9 \%)$ & $20(55.6 \%)$ & \multirow{3}{*}{$0.161^{*}$} \\
\hline & Enhancement & $8(25 \%)$ & $16(44.4 \%)$ & \\
\hline & Shadowing & $1(3.1 \%)$ & 0 & \\
\hline \multirow{2}{*}{ Calcifications } & In the mass & $3(9.4 \%)$ & $1(2.8 \%)$ & \multirow{2}{*}{$0.336^{+}$} \\
\hline & Absent & $20(90.6 \%)$ & $35(97.2 \%)$ & \\
\hline \multirow{2}{*}{ Distortion } & Present & 0 & 0 & \multirow{2}{*}{ - } \\
\hline & Absent & $32(100 \%)$ & $36(100 \%)$ & \\
\hline \multirow{2}{*}{ Ductal change } & Present & $1(3.1 \%)$ & $3(8.3 \%)$ & \multirow{2}{*}{$0.616^{+}$} \\
\hline & Absent & $31(96.9 \%)$ & $33(91.7 \%)-$ & \\
\hline \multirow[t]{2}{*}{ Skin change } & Present & 0 & 0 & \multirow{2}{*}{-} \\
\hline & Absent & $32(100 \%)$ & $36(100 \%)$ & \\
\hline Edema & Present & 0 & 0 & - \\
\hline & Absent & $32(100 \%)$ & $36(100 \%)$ & - \\
\hline
\end{tabular}

* Chi-square test

+ Fisher's exact test 